\title{
Validade e fidedignidade da Escala de Depressão Geriátrica na identificação de idosos deprimidos em um hospital geral
}

\author{
Validity and reliability of the Geriatric Depression Scale for the \\ identification of depressed patients in a general hospital
}

Rilva Lopes de Sousa', José Givaldo Melquíades de Medeiros', Ana Carolina Leite de Moura', Clarissa Lima e Moura de Souza', Iramirton Figuerêdo Moreira?

\section{RESUMO}

Objetivo: $\bigcirc$ objetivo deste estudo foi avaliar validade e fidedignidade da Escala de Depressão Geriátrica (EDG) nas versões de 30 e 15 itens na identificação de suspeitas de depressão em idosos nas enfermarias de clínica médica do Hospital Universitário Lauro Wanderley, em João Pessoa, Paraíba. Métodos: Realizou-se um estudo observacional e transversal, com aplicação da EDG-30 e da EDG-15 por uma entrevistadora treinada, efetuando-se no mesmo dia exame mental dos pacientes por um psiquiatra de acordo com critérios da CID-10. A fidedignidade das duas versões da EDG foi avaliada por meio da técnica do teste-reteste e da equivalência interobservadores. Resultados: A concordância entre a aplicação da EDG30 e o exame psiquiátrico foi significativa e moderada ( $k a p p a=0,48 ; p=0,04$ ), enquanto a EDG-15 não apresentou concordância estatística ( $p=0,62$ ). A EDG-30 apresentou sensibilidade de $83 \%$ e especificidade de 57\%, mas a EDG-15 apresentou sensibilidade de 50\% e especificidade de 62\%. Os escores da EDG-30 apresentaram correlação estatisticamente significativa entre si no teste-reteste e na equivalência entre observadores, porém a versão de 15 itens não foi fidedigna. Conclusões: Conclui-se que a EDG-30 é mais sensível e fidedigna que a EDG-15. Assim, a EDG-30 é útil para detectar como negativos os pacientes que realmente não apresentem depressão, porém são necessários estudos posteriores para adequação dos itens dessa escala à nossa clientela, a fim de aumentar sua especificidade e valor preditivo positivo.

Saúde do idoso, depressão, EDG.

\begin{abstract}
Objective: The aim of this study was to evaluate the validity and reliability of the Geriatric Depression Scale (GDS) in the versions of 30 and 15 items for the identification of suspicion of depression in the elderly in the infirmary of general practice of the Hospital Universitário Lauro Wanderley, in João Pessoa, Paraiba. Methods: An observational and transversal study, with the application of GDS-30 and GDS-15 by a trained interviewer, was held at the same day the mental exam of the patients by a psychiatrist, according to the criteria of ICD-10. The concordance of the
\end{abstract}

1 Universidade Federal da Paraíba (UFPB). 


\section{Key-words}

Old age health, depression, GDS. two versions of the GDS was evaluated by the test-retest method and inter-observers equivalence. Results: The concordance between the application of the GDS-30 and the psychiatric exam was significant and moderated (kappa $=0.48, p=0.04$ ), while the GDS-15 didn't present statistics concordance $(p=0.62$ ). The GDS-30 presented sensitivity of $83 \%$ and specificity of $57 \%$, but the GDS-15 presented sensitivity of 50\% and specificity of 62\%. Scores of GDS-30 presented significant statistically correlation in the test-retest method and inter-observers equivalence, but the version of 15 items weren't reliable. Conclusions: The conclusion was that the GDS-30 is more sensitive and reliable than the GDS-15, however its sensitivity was inferior to the numbers related in other studies, while his specificity was not high. Therefore, the GDS-30 is useful to detect as negatives the patients who really do not have depression, however further studies are necessary to adjust the items of this scale to our clientele, in order to enhance its specificity and positive predictive value.

\section{INTRODUÇÃO}

A depressão tem uma prevalência de 35\% a 45\% entre idosos hospitalizados em enfermarias de clínica médica geral (Maleiro, 2000; Almeida e Almeida, 1999), representando um problema de grande repercussão sobre mortalidade e evolução hospitalar desses pacientes (Furlanetto et al., 1998).

Apesar de sua relevância clínica, é baixa a detecção de sintomatologia depressiva em idosos nesse contexto, o que pode ser atribuído, em parte, à expressão clínica diferente desses sintomas em relação aos demais grupos etários. Há também uma baixa valorização dessas manifestações por parte dos médicos generalistas, que geralmente focalizam sua atenção clínica na doença que motiva a internação do paciente (Sousa et al., 2001). Nesse sentido, a avaliação sistemática dos pacientes idosos mediante a aplicação de escalas de avaliação de sintomas depressivos por médicos generalistas poderia contribuir para melhorar a detecção dos casos de depressão nesse grupo etário em clínica médica, onde representa a maior parte das hospitalizações.

A Escala de Depressão Geriátrica (EDG) constitui o instrumento mais empregado para avaliar sintomas depressivos em populações geriátricas, sendo usada em pesquisa e em contextos clínicos (Blank et al., 2004). Criada por Yesavage et al. (1983), a EDG passou a ser considerada uma escala com propriedades de validade e confiabilidade satisfatórias para rastreamento de depressão no idoso (Ertan e Eker, 2000; Hoyl et al., 1999), sendo traduzida para o português e adaptada para aplicação no Brasil por Stoppe Júnior et al. (1994).

Vários estudos foram realizados posteriormente no Brasil, empregando-se a tradução para o português da EDG em duas versões (30 e 15 itens) em diferentes contextos clínicos em São Paulo, tanto para idosos atendidos ambulatorialmente (Almeida e Almeida, 1999; Ribeiro et al., 1994) quanto em enfermarias de geriatria (Mascarenhas et al., 1996). No entanto, a tradução e validação realizadas na Região Sudeste podem não ser adequadas para aplicação em estados da Região Nordeste, por isso o objetivo do presente estudo foi avaliar a adequação da EDG em duas versões (EDG-30 e EDG-15) para identificação de suspeitas de depressão em idosos atendidos nas enfermarias de clínica médica do Hospital Universitário Lauro Wanderley, em João Pessoa (PB).

\section{MÉTODOS}

O presente estudo seguiu um delineamento observacional e transversal a partir de entrevistas estruturadas com 50 pacientes idosos consecutivamente internados nas enfermarias de clínica médica do HULW. Incluíram-se pacientes com idade igual ou superior a 60 anos, excluindo-se aqueles doentes com impossibilidade de comunicação, em estado clínico explicitamente grave e com queixa corrente de dor.

Os principais conceitos psicométricos utilizados na análise foram os de validade e confiabilidade. Validade diz respeito à capacidade de um instrumento em medir aquilo a que se propõe medir, ou seja, é o grau em que um instrumento discrimina entre pessoas que diferem em determinada característica de acordo com um critério-padrão chamado de padrão-ouro (Kelsey, 1996). Confiabilidade, ou fidedignidade, refere-se ao grau de concordância entre múltiplas medidas de uma variável, isto é, o "grau de concordância entre múltiplas medidas de um mesmo objeto" (Menezes, 1998).

Para avaliar a concordância entre as versões da EDG, aplicaram-se a EDG 30 e a EDG-15 aos pacientes internados na mesma sessão por uma entrevistadora treinada (entrevistadora I). Para avaliação da equivalência interobservadores, repetiu-se após 1 semana a aplicação por outra entrevistadora (entrevistadora II), a qual não tinha conhecimento dos resultados da aplicação prévia.

Para estimativa da validade de critério, realizaram-se entrevistas por um psiquiatra no dia da segunda aplicação das escalas, seguindo-se os critérios diagnósticos da Classificação Internacional de Doenças Mentais, 1̄e edição, CID-10 
(OMS, 1993). Nessa avaliação, utilizando-se como padrãoouro o diagnóstico psiquiátrico, analisaram-se a sensibilidade e a especificidade das duas versões da escala administradas pela entrevistadora II. Para a EDG-30, adotou-se o ponto de corte de 10, enquanto para a EDG-15 admitiu-se o ponto de corte de 5 , de acordo com os estudos de validação da escala em São Paulo (Almeida e Almeida, 1999; Stoppe Júnior et al., 1994). Um escore alto obtido pelo idoso na aplicação dessa escala, com base nesses pontos de corte, indica a presença de sintomas depressivos clinicamente significativos e suspeita de depressão a ser confirmada, ou não, pelo exame psiquiátrico.

De acordo com Fletcher et al. (1989), calcularam-se a sensibilidade por meio da fórmula $a / a+c$ e a especificidade como d/b+d, sendo a chance de concordância (teste Kappa) expressa pela fórmula: concordância observada - concordância esperada/100 - concordância observada. Estudaram-se os dados por meio da análise de correlação linear simples de Speraman, do Teste de Concordância ou Kappa e de comparação de médias pelo Teste t de Student. O projeto do estudo recebeu parecer favorável do Comitê de Ética em Pesquisa do HULW/UFPB (processo número 331/04). Os pacientes elegíveis foram incluídos no estudo após assinar o termo de consentimento livre e esclarecido.

\section{RESULTADOS}

Na caracterização sociodemográfica da amostra, observouse que a idade variou de 60 a 85 anos $(71,1 \pm 6,02)$, sendo $52 \%$ do sexo masculino, $60 \%$ casados e $56 \%$ procedentes de cidades do interior da Paraíba (Tabela 1). A profissão prevalente foi a de agricultor (44\% da amostra), seguida pela função "do lar" (22\%).

Tabela 1. Caracterização sociodemográfica da amostra $(n=50)$

\begin{tabular}{lccc}
\hline & \multicolumn{2}{c}{ Variáveis } & \multicolumn{2}{c}{ Freqüências } \\
\cline { 3 - 4 } & & $f$ & $\%$ \\
\hline Faixa etária & $60-69$ & 24 & 48 \\
& $70-79$ & 23 & 46 \\
Sexo & 80 ou mais & 3 & 6 \\
& Masculino & 22 & 52 \\
Estado civil & Feminino & 24 & 48 \\
& Casados & 30 & 60 \\
Procedência & Não-casados & 20 & 40 \\
& Capital & 22 & 44 \\
\hline
\end{tabular}

Houve perda de quatro pacientes ( $0,8 \%$ da amostra) pela ocorrência de alta hospitalar ou transferência dos pacientes inicialmente incluídos na pesquisa. Portanto, da amostra de 50 pacientes selecionada primeiramente e considerada pela entrevistadora I, 46 foram também avaliados pela en- trevistadora II e, por isso, apresentam as duas entrevistas, sendo, portanto, este o número de pares avaliado para efeito de análise de correlação interobservadores e para estudo da validade em relação ao diagnóstico psiquiátrico. Dos 46 idosos abordados pelas duas entrevistadoras, sete pacientes (14\% dos avaliados) receberam diagnóstico de depressão pelo psiquiatra. Por meio da aplicação da EDG-30, a entrevistadora I detectou $22(47,8 \%)$ e a entrevistadora II identificou 25 (54,3\%) pacientes acima do ponto de corte de 10 pontos. Com a administração da EDG-15, as freqüências de alcance do ponto de corte de 5 foram de $18(39,1 \%)$ e de 15 $(32,6 \%)$ para a entrevistadora I e a II, respectivamente.

A figura 1 mostra a distribuição dos escores médios da EDG-30 de acordo com os intervalos de idade em homens e mulheres. Observou-se que os escores médios das muIheres foram mais altos que os dos homens nas faixas etárias de 60 a 69 anos e de 70 a 79 anos, porém, acima de 80 anos, as pontuações médias tendem a aproximar-se, inclusive com a superposição dos escores e ligeira superioridade dos homens, mas essa diferença não alcançou significância estatística. Com a aplicação da escala abreviada, não se observaram diferenças de gênero.

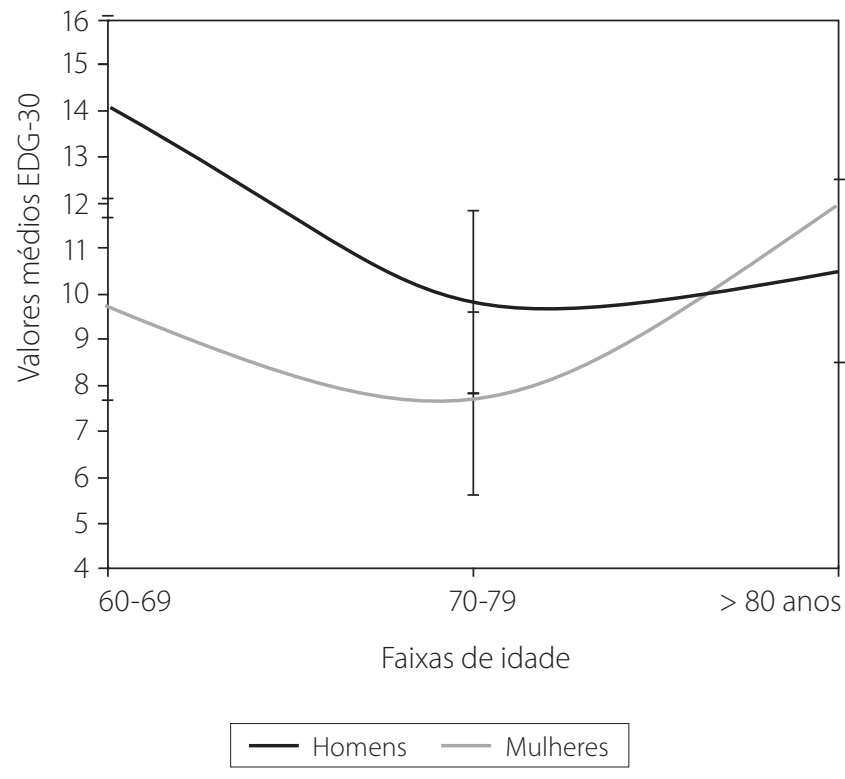

Figura 1. Distribuição dos valores médios da EDG-30 (Escala de depressão geriátrica, 30 itens) entre homens e mulheres, de acordo com categorias de idade $(60-69,70-79$, mais de 80 anos).

Quanto à confiabilidade entre as duas aplicações da mesma versão, verificou-se que os escores da EDG-30 obtidos pela entrevistadora I apresentaram correlação significativa estatisticamente (EDG 30-I e EDG 30-II: rs = 0,60; $p=$ 0,01), enquanto os da EDG-15 não apresentaram correlação (EDG 15-I e EDG 15-II: $p=0,18=N S$ ). Os escores obtidos 
pela entrevistadora II apresentaram resultados semelhantes. O coeficiente de correlação entre as duas aplicações por entrevistadoras independentes revelou que a EDG 30 e a EDG 15 apresentaram correlação significativa entre si, sendo de $82 \%$ ( $r s=0,82 ; p=0,01)$ e $79 \%$ ( $r s=0,79 ; p=0,03$, respectivamente.

Quanto à validade, a concordância entre o resultado da aplicação da EDG-30 e o exame psiquiátrico foi estatisticamente significativa, com magnitude de 48\% ( $p=0,04$, kappa $=0,48$ ), sendo essa concordância de moderada intensidade, enquanto para a EDG-15 não houve concordância significativa ( $p=0,52=$ NS). Calculando-se, então, os índices preditivos, a partir da sensibilidade, especificidade e prevalência de depressão na amostra, verificaram-se baixos índices preditivos positivos e altos índices preditivos negativos, conforme demonstra a tabela 2.

Tabela 2. Validade de critério das aplicações da Escala de Depressão Geriátrica (EDG: EDG-30 e EDG-15) por duas entrevistadoras independentes com base no diagnóstico psiquiátrico (CID-10)

\begin{tabular}{lcccc}
\hline \multirow{2}{*}{ Propriedades } & \multicolumn{4}{c}{ Escalas } \\
\cline { 2 - 5 } & \multicolumn{2}{c}{ EDG-30 } & \multicolumn{2}{c}{ EDG-15 } \\
\cline { 2 - 5 } Sensibilidade & Entrev. I & Entrev. II & Entrev. I & Entrev. II \\
\hline Especificidade & $83 \%$ & $63 \%$ & $50 \%$ & $16 \%$ \\
Valor preditivo positivo & $62 \%$ & $65 \%$ & $57 \%$ & $47 \%$ \\
Valor preditivo negativo & $95 \%$ & $16 \%$ & $16 \%$ & $6 \%$ \\
\hline
\end{tabular}

\section{DISCUSSÃO}

A EDG tem sido empregada para avaliação de sintomas depressivos em idosos na comunidade, mas também em pacientes idosos ambulatoriais (Garcia-Serrano e Tobias-Ferrer, 2001; Hernandéz e Mocetzuma, 1999), em asilos (Koehler et al., 2005; Mossey e Gallagher, 2004) e em idosos internados em enfermarias gerais (Schüller et al., 2004; Covinsky et al., 1997).

Na nossa amostra em enfermarias de clínica médica, a prevalência de depressão nessa amostra (14\%) foi menor que as taxas publicadas na literatura, as quais são de $35 \%$ a 45\% para idosos hospitalizados em enfermarias de clínica geral. Contudo, no presente estudo foram excluídos os pacientes com dor e doenças graves. Sabe-se que a dor está significativamente associada à depressão e aos sintomas depressivos em idosos (Mossey e Gallagher, 2004; Schüller et al., 2004). Com a aplicação das escalas, verificou-se, entretanto, que $47,8 \%$ a $54,3 \%$ dos pacientes atingiram o ponto de corte na EDG-30 e 32\% a 39\% dos pacientes na EDG-15.

Nesse sentido, em pacientes idosos é importante salientar os termos depressão "subliminar" e "subclínica", a chamada "depressão subsindrômica" (Blank et al., 2004). Essa terminologia tem sido usada na literatura para designar sintomas depressivos que não preenchem completamente o quadro de um episódio depressivo, já que não há sintomas suficientes, nem são sintomas suficientemente intensos, mas que implicam algum grau de sofrimento para as pessoas afetadas (Banger, 2002). Recentemente, particular atenção tem sido dada aos pacientes com esses quadros sintomáticos leves que não preenchem critérios diagnósticos para depressão maior (Brown e Solchany, 2004). Hybels et al. (2001) consideram que sendo a prevalência de depressão elevada entre pacientes idosos e sua apresentação clínica atípica nessa faixa de idade, é necessário excluir os casos de depressão subsindrômica nessa clientela.

O encontro de pontuações mais altas em mulheres até a faixa etária de 80 anos é um dado esperado, sendo este um aspecto relatado em outros trabalhos, nos quais as mulheres tendem a apresentar menor dificuldade de admitir a presença de sintomas psicológicos que os homens (Kessler, 2000). Além disso, as mulheres são mais vulneráveis ao desenvolvimento de transtornos depressivos na velhice (Reynolds et al., 1999; Furlanetto et al., 1998), contribuindo para isso a alta taxa de viuvez e de isolamento social entre as mulheres e também a insuficiência ovariana após a menopausa (Sousa et al., 2000).

Considera-se ainda que, existindo uma diferença de sintomatologia depressiva em relação ao gênero, essa discrepância reduz-se substancialmente na idade mais avançada quando passa a haver uma equivalência (George, 1999), o que é concordante com a distribuição dos escores entre homens e mulheres de acordo com a faixa de idade observada aqui. As taxas de prevalência para depressão maior também apresentam uma distribuição similar à de sintomatologia depressiva. Noble (2005) cita resultados de estudos epidemiológicos que revelam prevalência de 21,3\% de depressão maior em mulheres, quase duas vezes maior que nos homens $(12,7 \%)$, mas essas diferenças de prevalência em função do gênero também tendem a desaparecer depois da meia-idade.

Quanto à confiabilidade entre as duas aplicações da mesma versão, verificou-se que os escores da EDG-30 apresentaram correlação estatisticamente significativa entre si, de sentido positivo e de moderada intensidade, corroborando os dados de Ertan e Eker (2000), no estudo de validação da EDG-30 na Turquia, onde esses autores encontraram um índice de correlação de 0,74 na aplicação da escala por dois entrevistadores independentes.

A sensibilidade da EDG-30 variou de 63\% a 83\% nas duas aplicações, enquanto a sua especificidade variou de $62 \%$ a $65 \%$. Esses índices foram inferiores aos relatados pelo autor da escala, que constatou sensibilidade de 95\% e especificidade de 84\% para a EDG-30 (Yesavage et al., 1983), e também em relação ao estudo de validação no Brasil (Stoppe Júnior 
et al., 1994), com o mesmo ponto de corte de 10, evidenciando-se sensibilidade de $92 \%$ e especificidade de $89 \%$. A análise da concordância entre EDG-30 e exame psiquiátrico foi de $48 \%$, uma concordância de moderada intensidade, enquanto para a EDG-15 não houve concordância com o exame psiquiátrico. A concordância encontrada na aplicação da EDG-30 assemelha-se apenas à taxa de 46\% verificada por Greenberg et al. (2004) na aplicação da EDG-30 em pacientes idosos institucionalizados nos Estados Unidos.

Quanto à versão de 15 itens da EDG, não se verificou concordância com o diagnóstico psiquiátrico no presente estudo. Almeida e Almeida (1999) demonstraram que a versão brasileira do EDG-15 oferece medida válida para o diagnóstico de episódio depressivo e o ponto de corte 5/6 (não caso/caso) produz índice de sensibilidade de 85,4\% e especificidade de 73,9\% para o diagnóstico desses episódios.

A EDG-15, que também apresentou validade insatisfatória e correlação interobservadores ausente no presente estudo, é recomendada na Inglaterra como triagem de depressão em idosos na comunidade (Osborn et al., 2002). No entanto, a clientela de idosos internados em enfermarias de clínica médica é distinta de idosos avaliados em seus domicílios, ou mesmo em serviços de atenção primária, embora tenha sido empregada em estudos clínicos (Ribeiro et al., 2004; Almeida e Almeida, 1999). Nesse sentido, Edwards (2004) pondera que o uso da EDG em amostras clínicas deve adotar um ponto de corte mais baixo. Além disso, alguns itens da EDG cujas "respostas depressivas" seriam as afirmativas ("sim") deveriam, na verdade, ser indicativos de "normalidade" no contexto clínico de uma hospitalização. Por exemplo, dois dos itens que fazem parte da EDG-15: "O senhor acha ruim o modo como se encontra agora?" (item 12) e "O senhor se sente cheio de energia?" (item 13).

A diferença entre o resultado da validade da EDG-30 e da EDG-15 pode ser atribuída, em parte, às diferenças devidas à amostragem de itens. NA EDG-15, avaliam-se sintomas de insatisfação com a vida, sensação de vida vazia, sentimento de desesperança, sensação de desamparo, medo de maus acontecimentos, retraimento social e perda de energia. Na versão abreviada, não são abordados os seguintes itens contidos na EDG-30: diminuição do interesse e prazer, sentimento de tédio, sensação de inutilidade, vontade freqüente de chorar, sensação de culpa, sensação de fadiga ao despertar, desânimo, agitação, tristeza, baixa auto-estima, dificuldades de tomar decisões e redução da capacidade mental. Esses sintomas, que não são abordados na EDG-15, provavelmente fizeram a diferença na capacidade de a escala abreviada detectar casos de pacientes deprimidos.

Nesse sentido, Adams et al. (2004) realizaram análise fatorial da EDG-30, chegando à conclusão de que a presença de perguntas cobrindo pelo menos quatro subdimensões, quais sejam humor disfórico, apatia, alteração cognitiva e ansiedade, melhora a precisão e a utilidade da escala para identificar casos de depressão. Contudo, os sintomas em comum às duas escalas foram suficientes para a obtenção de um coeficiente de correlação satisfatório entre as duas versões. Os baixos índices preditivos positivos e altos índices preditivos negativos indicam que a EDG-30 é útil em detectar como negativos os pacientes que realmente não apresentam depressão nessa amostra, servindo, portanto, nessa clientela para excluir os pacientes não-deprimidos, deixando, contudo, de detectar os casos de doentes deprimidos.

Consideramos relevante também levar em conta que a validade de um instrumento para avaliação de sintomas depende primordialmente do nível de instrução e da compreensão dos itens para os entrevistados, por isso esses questionários precisam ser avaliados em cada cultura e posteriormente comparados por meio de estudos em diferentes populações. É necessário, sobretudo, levar em consideração as grandes diferenças culturais que existem mesmo entre regiões do Brasil. A tradução e a validação realizadas na Região Sudeste do país podem realmente não ser adequadas para aplicação na Paraíba, sobretudo em uma clientela de nível socioeducacional desfavorável.

\section{CONCLUSÕES}

Concluímos que a EDG-30 é mais sensível e fidedigna que a EDG-15, porém sua sensibilidade foi inferior aos índices relatados em outros estudos, e sua especificidade também não foi alta como nas publicações feitas na Região Sudeste; deduzimos também que a EDG pode ser útil para detectar como negativos os pacientes que realmente não apresentem depressão, mas são necessários estudos posteriores para adequação dos itens dessa escala à nossa clientela, para aumentar a sua especificidade e valor preditivo positivo.

\section{REFERÊNCIAS}

Adams KB, Matto HC, Sanders S. Confirmatory factor analysis of the geriatric depression scale. Gerontologist, 44:818-26, 2004.

Almeida OP, Almeida SA. Confiabilidade da versão brasileira da Escala de Depressão Geriátrica (GDS) versão reduzida. Arq Neuropsiquiatr, 57:421-6, 1999

Banger M. Affective syndrome during perimenopause. Maturitas, 41:S13-8, 2002.

Blank K, Gruman C, Robison JT. Case-finding for depression in elderly people: balancing ease of administration with validity in varied treatment settings. J Gerontol A Biol Sci Med Sci, 59:378-84, 2004.

Brown, MA, Solchany JE. Two overlooked mood disorders in women: subsyndromal depression and prenatal depression. Nurs Clin North Am 39 (1):83-95, 2004

Covinsky KE et al. Relation between symptoms of depression and health status outcomes in actwly ill hospitalized older persons. Ann Inter Med, 127:1131-2, 1997.

Edwards M. Assessing for depression and mood disturbance in later life. Br J Community Nurs, 9:492-4, 2004 
Ertan T, Eker E. Reliability, validity, and factor structure of the geriatric depression scale in Turkish elderly: are there different factor structures for different cultures? Int Psychogeriatr, 12:163-72, 2000

Fletcher RH, Fletcher SW, Wagner EH. Diagnóstico. In: Fletcher RH, Fletcher SW, Wagner EH. Epidemiologia clínica. Porto Alegre: Artes Médicas p. 68-107, 1989.

Furlanetto LM, Bueno JR, Silva RV. Características e evolução de pacientes com transtornos depressivos durante a internação em enfermarias de clínica médica. Jornal Brasileiro de Psiquiatria, 47:609-17, 1998.

Garcia Serrano MJ, Tobias Ferrer J. Prevalence of depression in patients over 65 years of age. Profile of the elderly at risk. Rev Aten Primaria, 27:484-8, 2001.

George LK. Fatores sociais e econômicos relacionados aos transtornos psiquiátricos do idoso. In: Busse EW, Blazer DG. Psiquiatria geriátrica. Porto Alegre: Artes Médicas p. 146, 1999.

Greenberg L, Lantz MS, Likourezos A. Screening for depression in nursing home palliative care patients. J Geriatr Psychiatry Neurol, 17:212-8, 2004.

Hernandéz SPS, Mocetzuma LEA. Depresión en población adulta mayor: tamizage en unidade de primer nivel de atención médica. Rev Med IMSS, 37:111-5, 1999.

Hoyl MT et al. Development and testing of a five-item version of Geriatric Depression Scale. Journal of the American Geriatrics Society, 47:873-8, 1999.

Hybels CF, Blazer DG, Pieper CF. Toward a threshold for subthreshold depression: an analysis of correlates of depression by severity of symptoms using data from an elderly community sample. Gerontologist, 41:357-65, 2001

Kelsey, JE. Dose-response relationship with venlafaxine. J Clin Psychopharmacol, 16 (3 Suppl 2):215-65, 1996

Kessler RC. Psychiatric epidemiology: selected recent advances and future directions. Bull World Health Organ, 78:464-74, 2000.

Koehler M, Rabinowitz T, Hirdes J. Measuring depression in nursing home residents with the MDS and GDS: an observational psychometric study. BMC Geriatr, 5:1-3, 2005.

Maleiro AMAS. Tratamento da depressão leve a moderada: avaliação da eficácia e segurança do extrato de Hypericum perforatum. Rev Bras Med, 57:1313-23, 2000.

Mascarenhas AN, Moraes ML, Guimarães RM. Depressão em idoso: elevada prevalência e baixa detecção. Gerontologia, 4:30-7, 1996.
Menezes, PR. Validade e confiabilidade de escalas de avaliação em psiquiatria. Rev Psiq Clin, 25 (5):214-6, 1998 .

Mossey JM, Gallagher RM. The longitudinal occurrence and impact of comorbid chronic pain and chronic depression over two years in continuing care retirement community residents. Pain Med, 5:335-48, 2004

Noble RE. Depression in women. Metabolism, 54:49-52, 2005.

Osborn DP et al. Geriatric depression scale in a representative sample of 14.545 people aged 75 and over in the United Kingdom: results from the MRC Trial of Assessment and Management of Older People in the Community. Int J Geriatr Psychiatry 17(4):375-82, 2002

Organização Mundial da Saúde. Classificação de transtornos mentais e de comportamento da CID-10: descrições clínicas e diretrizes diagnósticas. Trad. Dorgival Caetano. Porto Alegre: Artes Médicas p. 5-14, 1993.

Reynolds (F et al. Behavioral and pharmacologic interventions for depression in later life. In: Schulz M, Lawton MP. Annual Review of Gerontology and Geriatrics. New York: Springer Publishing Company p. 48-71, 1999.

Ribeiro JEC et al. Associação entre aspectos depressivos e déficit visual causado por catarata em pacientes idosos. Arq Bras Oftalmol, 67:795-9, 2004.

Ribeiro MAA et al. Prevalência da depressão em idosos institucionalizados em tempo integral. Rev Psiquiat Clín, 21:4-8, 1994.

Schüler M et al. Acute and chronic pain in geriatrics: clinical characteristics of pain and the influence of cognition. Pain Med, 5:253-62, 2004

Sousa RL. Ensaio clínico placebo-controlado com reposição hormonal para sintomas depressivos e somáticos em mulheres no climatério. Dissertação de mestrado (Desenvolvimento Humano). Universidade Federal da Paraíba, João Pessoa, 2000. Stoppe Júnior A, Jacob Filho W, Louzã Neto MR. Avaliação de depressão em idosos através da Escala de Depressão em Geriatria: resultados preliminares. Rev. ABP-APAL, 16:149-53, 1994.

Sousa RL et al. Valorização de sintomas depressivos em idosos internados em enfermarias de clínica médica. Revista Brasileira de Clínica \& Terapêutica, 27:183-8, 2001.

Yesavage JA et al. Development and validation of a geriatric screening scale. Journal of Psychiatry Research, 17:37-49, 1983. 